\title{
Convexity ranks in higher dimensions
}

\author{
by
}

\section{Menachem Kojman (Beer Sheva)}

\begin{abstract}
A subset of a vector space is called countably convex if it is a countable union of convex sets. Classification of countably convex subsets of topological vector spaces is addressed in this paper.

An ordinal-valued rank function $\varrho$ is introduced to measure the complexity of local nonconvexity points in subsets of topological vector spaces. Then $\varrho$ is used to give a necessary and sufficient condition for countable convexity of closed sets.

Theorem. Suppose that $S$ is a closed subset of a Polish linear space. Then $S$ is countably convex if and only if there exists $\alpha<\omega_{1}$ so that $\varrho(x)<\alpha$ for all $x \in S$.

Classification of countably convex closed subsets of Polish linear spaces follows then easily. A similar classification (by a different rank function) was previously known for closed subset of $\mathbb{R}^{2}[3]$.

As an application of $\varrho$ to Banach space geometry, it is proved that for every $\alpha<\omega_{1}$, the unit sphere of $C(\omega \alpha)$ with the sup-norm has rank $\alpha$. Furthermore, a countable compact metric space $K$ is determined by the rank of the unit sphere of $C(K)$ with the natural sup-norm:

THEOREM. If $K_{1}, K_{1}$ are countable compact metric spaces and $S_{i}$ is the unit sphere in $C\left(K_{i}\right)$ with the sup-norm, $i=1,2$, then $\varrho\left(S_{1}\right)=\varrho\left(S_{2}\right)$ if and only if $K_{1}$ and $K_{2}$ are homeomorphic.
\end{abstract}

Uncountably convex closed sets are also studied in dimension $n>2$ and are seen to be drastically more complicated than uncountably convex closed subsets of $\mathbb{R}^{2}$.

1. Introduction. Suppose that $S$ is a set in a linear space, and $S$ is not convex. How far is $S$ from being convex?

The set $A$ in Figure 1 is more convex than the set $B$, because $A$ is a union of two convex sets, whereas $B$ is a union of three, but not two, convex sets.

2000 Mathematics Subject Classification: Primary 52A07, 04A15; Secondary 03E05, $04 \mathrm{~A} 30$.

Key words and phrases: convexity, convexity number, Polish vector space, continuum hypothesis, Cantor-Bendixson degree. 


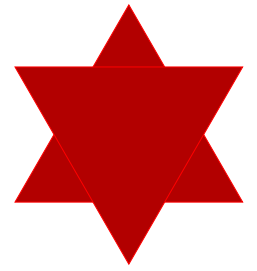

A

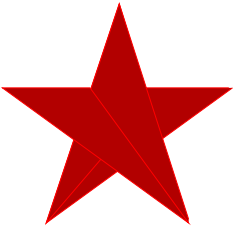

B

Fig. 1

Let the convexity number $\gamma(S)$ of a set $S$ be the least number $\gamma$ so that $S$ is a union of $\gamma$ convex sets.

There are two general problems concerning convexity numbers. One is computing convexity numbers of given sets. The other is classifying all types of sets with a fixed convexity number.

The standard approach to classification of sets with a given convexity number has been by studying sizes of visually independent subsets of the set. A subset $V \subseteq S$ is visually independent in $S$ if no two points in $S$ see each other through $S$. Let $\alpha(S)$ be the supremum of cardinalities of visually independent subsets of $S$. It is always true that $\alpha(S) \leq \gamma(S)$.

The pentagonal star above is an example of a set with $\alpha=2$ and $\gamma=3$. Thus there are two $\alpha$-types of sets with $\gamma=3: \alpha=2$ and $\alpha=3$.

Valentine [20] proved that if $S \subseteq \mathbb{R}^{2}$ is closed and $\alpha(S)=2$, then $\gamma(S)$ $\leq 3$. Therefore, there are exactly two $\alpha$-types of closed planar sets with $\gamma=4(\alpha=3$ and $\alpha=4)$, as $\alpha=2$ is ruled out by Valentine's Theorem.

A long line of results concerning the relation between $\alpha$ and $\gamma$ in closed planar sets followed Valentine's result $[6,14,2,1,3,19,17]$. The most recent advance in determining all types of closed planar sets with $\gamma(S)=n$ is the result by Matoušek and Valtr in [17]: if $\alpha=n$ then $\gamma \leq 18 n^{3}$ and examples are provided of sets with $\alpha=n$ and $\gamma \geq O\left(n^{2}\right)$. The exact number of different $\alpha$-types of closed planar sets with $\gamma(S)=n$ ranges between $n-O\left(n^{1 / 2}\right)$ and $n-O\left(n^{1 / 3}\right)$.

Sets with infinite $\gamma$, which are the subject of the present paper, were first studied in [13]. It turned out that although visually independent subsets fail to capture uncountability of $\gamma$ in closed planar sets, certain generalizations of visually independent sets do: a subset of $Q \subseteq S$ is called a 3-clique if for every 3-element set $X \subseteq Q$, the convex hull of $X$ is not contained in $S$. Visually independent sets are, in this terminology, 2-cliques. A closed planar set with $\gamma(S)>\aleph_{0}$ need not contain an uncountable 2-clique, but must contain a perfect, nonempty (hence uncountable) 3-clique.

Cardinalities of cliques do not distinguish between one set with $\gamma=\aleph_{0}$ and another. Topological degrees of 3-cliques in a countably convex set, do, 
however, serve the purpose of classification of countable convexity. In [12] it is proved that there is a uniform bound $\delta(S)$ over all Cantor-Bendixson degrees of 3-cliques in a given closed planar $S$ with $\gamma(S)=\aleph_{0}$. Classifying by $\delta$, one obtains $\omega_{1}$ different $\delta$-types of closed, planar, countably convex sets.

It is of course desirable to extend classification of $\gamma$ by clique properties to sets outside the class of closed planar sets. But there are certain a priori limitations: The convexity number of a set is always equal to the chromatic number of the set's convexity hypergraph (see 3 below), in which 2-, 3- and $n$-cliques are combinatorial cliques. In general, the clique number of a hypergraph is poorly related to the hypergraph's chromatic number. Convexity hypergraphs of closed planar sets are well behaved in the sense that they have a large chromatic number only when they have a large clique number. However, closed sets in $\mathbb{R}^{3}$ may have uncountable $\gamma$ but only countable cliques, and in $\mathbb{R}^{4}$ every graph can be realized as the convexity graph of some set, so, for example, in [13] a subset of $\mathbb{R}^{4}$ is given with $\alpha(S)=3$ and $\gamma(S)=2^{\aleph_{0}}$.

To avoid pathological hypergraphs, some limitations on the topological complexity of the set and/or the dimension of the space have to be imposed.

In the present paper classification of countably convex sets is extended to closed sets in Polish vector spaces. Classification is obtained by considering a generalization of perfect $n$-cliques, called semi-cliques .

Classification of closed sets by semi-cliques is sharp in the sense that semi-cliques fail to determine $\gamma$ already for $F_{\sigma}$ and $G_{\delta}$ subsets of $\mathbb{R}^{2}$.

An application of $\varrho$ to Banach space geometry is given in Section 17, where it is proved that for every $\alpha<\omega_{1}$ there exists some Banach space in which the unit sphere $S$ has $\operatorname{rank} \varrho(S)=\alpha$, and that a countable metric compact space $K$ can be retrieved from the convexity degree of the unit sphere in $C(K)$, the space of all real continuous functions on $K$ with the sup norm.

Finally, some attention is given in Sections 5 and 6 to sets which are not countably convex. Section 7 addresses open problems.

Acknowledgments. It is my pleasant duty to thank my colleague Professor V. Fonf for generously sharing with me his knowledge of Banach space geometry in numerous discussions and for his suggestion to apply $\varrho$ to unit spheres in Banach spaces.

Preliminaries and notation. Let $S$ always denote a subset of some vector space $V$ over the field of real numbers.

Let $\operatorname{conv}(X)$ denote the convex hull of a set $X \subseteq E$. A finite subset $X$ of $S$ is defected in $S$ if $X \subseteq S$ and $\operatorname{conv}(X) \nsubseteq \subseteq$. Call a subset $Q \subseteq S$ with the property that every $n$-element subset of $Q$ is defected in $S$, an $n$-clique. A 2-clique in $S$ is also called a visually independent subset of $S$. 
Carathéodory's theorem will be used freely:

Theorem 1. If $S \subseteq \mathbb{R}^{n}$ and $x \in \operatorname{conv}(X)$ then there is a subset $Y \subseteq X$ so that $x \in \operatorname{conv}(Y)$ and $|Y| \leq n+1$.

A topological vector space is a vector space equipped with a topology with respect to which vector addition and scalar multiplication are continuous. A topology $\tau$ on a space $X$ is second countable if it has a countable basis, and is Polish if $(X, \tau)$ is homeomorphic to a complete, separable, metric space. A Polish linear space is a topological vector space in which the topology is Polish.

A point $x \in S$ where $S$ is contained in a topological vector space is called a point of local convexity if there is some open neighborhood $u \ni x$ so that $\operatorname{conv}(S \cap u) \subseteq S$. A point $x \in S$ is a point of local nonconvexity or an lnc point if it is not a point of local convexity. The set of all lnc points of a set $S$ is denoted by $\operatorname{lnc}(S)$.

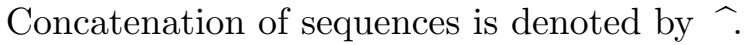

2. The convexity number is a chromatic number. This section is of a general nature. It is shown that the convexity number of a set is determined by the combinatorics of its finite defected subsets.

Let $S$ denote a subset of a linear space.

Definition 2. (i) Let $H=H(S)=\langle S, E\rangle$ be the hypergraph whose set of vertices is $S$ and whose set of hyperedges consists of all finite defected subsets of $S$.

(ii) Let $H_{n}(S)=\left\langle S, E \cap[S]^{n}\right\rangle$, that is, the $n$-regular hypergraph with set of vertices $S$ and whose set of edges consists of all defected $n$-element subsets of $S$.

(iii) A subset $F \subseteq S$ is free in $H(S)$ if no subset of $F$ is a hyperedge. The chromatic number of $H(S), \chi(H(S)$, is the least cardinality of a partition of $S$ into free sets.

TheOREM 3. For every set $S$ in a linear space $V$ :

(i) $\gamma(S)=\chi(H(S))$.

(ii) If $\operatorname{dim} V=n$ then $\gamma(S)=\chi\left(H_{n+1}(S)\right)$.

Proof. To prove (i) suppose $S=\bigcup_{\alpha<\lambda} C_{\alpha}$ where each $C_{\alpha}$ is convex. Let $D_{\alpha}=C_{\alpha}-\bigcup_{\beta<\alpha} C_{\alpha}$. Thus $\left\{D_{\alpha}: \alpha<\lambda\right.$ and $\left.D_{\alpha} \neq \emptyset\right\}$ is a partition of $S$. Since $D_{\alpha} \subseteq C_{\alpha}$ and the latter is convex, $D_{\alpha}$ is a free subset of $H(S)$ for all $\alpha<\lambda$.

Conversely, assume that $\chi(H(S))=\lambda$ and fix a partition $\left\{D_{\alpha}: \alpha<\lambda\right\}$ of $S$ so that each $D_{\alpha}$ is free. Let $C_{\alpha}=\operatorname{conv}\left(D_{\alpha}\right)$. Since no finite subset of $D_{\alpha}$ is defected, $C_{\alpha} \subseteq S$. Clearly, $S=\bigcup_{\alpha<\lambda} C_{\alpha}$. 
If $\operatorname{dim} V=n$ then by Carathéodory's theorem, a set $F \subseteq S$ is free in $H(S)$ iff it is free in $H_{n+1}(S)$. Hence (ii) holds.

Corollary 4 (Lawrence, Hare and Kenelly [14]). A set $S$ in a linear space is a union of $n$ convex sets if and only if every finite subset of $S$ is contained in the union of $n$ free sets in $H(S)$.

Proof. The fact that the chromatic number of a hypergraph is $\leq n$ iff the chromatic number of every finite subhypergraph is $\leq n$ is a well known Erdős-de Bruijn result (or, alternatively, a straightforward exercise in applying propositional calculus compactness). The rest is by Theorem 3 .

REMARK. The original proof in [14] makes use of Tikhonov's product theorem, which is equivalent to the full axiom of choice. However, only the axiom of choice for finite sets is needed for the theorem, since only propositional calculus compactness is required in the proof above.

3. Countable convexity and the radical of a set. Let $S$ be a subset of a second countable topological vector space. The first observation to be made on the way to determining whether $\gamma(S)$ is countable or not, is that a countable $\gamma(S)$ is a local property.

Definition 5. A point $x \in S$ is locally countably convex if there exists some open neighborhood $u \ni x$ so that $\gamma(S \cap u) \leq \aleph_{0}$.

FACT 6. Let $S$ be a subset of a second countable topological vector space. Let $A=A(S)$ be the set of all locally countably convex points of $S$ and let $B=B(S)=S-A$. Then:

(i) $A$ is relatively open in $S$ and $\gamma(S) \leq \aleph_{0}$.

(ii) $B$ is relatively perfect in $S$ (closed and without isolated points), and furthermore, for every $x \in B$ and open $u \ni x, B \cap u$ is uncountable.

(iii) If $x \in B$ and $u \ni x$ is open then there exist defected subsets of $B \cap u$.

(iv) $\gamma(S) \leq \aleph_{0}$ if and only if $B=\emptyset$.

Proof. (i) is immediate from the definition of $A$ and the fact that the topology is second countable. The fact that $B$ is closed follows from (i). Suppose that $x \in B$. If there exists a neighborhood $u \ni x$ so that $|B \cap u| \leq \aleph_{0}$ then $B \cap u$ is coverable by countably many singleton sets - each of which is trivially convex. Since $\gamma(A) \leq \aleph_{0}, \gamma(u \cap x) \leq \aleph_{0}$, contrary to $x \in B$. Thus (ii) follows. By similar reasoning, the points of $B \cap u$ are not contained in a single convex subset of $S$ for any $u$ so that $u \cap B \neq \emptyset$; therefore $\operatorname{conv}(B \cap u) \nsubseteq S$ for such $u$, and (iii) follows.

For the last item, if $B=\emptyset$ then $S=A$ and by (i), $\gamma(S) \leq \aleph_{0}$. Conversely, if $\gamma(S) \leq \aleph_{0}$ then clearly $B=\emptyset$. 
The fact above is a convexity analog of Cantor's structure theorem for sets in second countable spaces. Every set $S$ in a second countable linear space is the union of a countably convex set $A(S)$ and a relatively perfect set $B(S)$, where the latter is either null or not coverable by countably many convex subsets of $S$. (One should be cautious to observe that a subset of $B(S)$ which is defected in $B(S)$ need not necessarily be defected in $S$.)

What this fact does not give is a transfinite definition of $B(S)$, or, equivalently, a degree of countable convexity.

The next thing we seek is a degree function that relates to convexity numbers in the same way that Cantor's degree relates to cardinalities of sets: namely, which characterizes uncountably convex sets as those of unbounded degree and which classifies the countably convex ones.

The natural approach would be to define the rank as follows: Let $S^{0}=S$, let $S^{\alpha+1}=\operatorname{lnc}\left(S^{\alpha}\right)$, and for limit $\alpha$ let $S^{\alpha}=\bigcap_{\beta<\alpha} S^{\beta}$, that is, the derivative of a set is its subset of lnc points. This transfinite process must stabilize at some countable stage, and the remaining set is "perfect" with respect to local nonconvexity. However, it may be strictly bigger than $B(S)$, as in the following example.

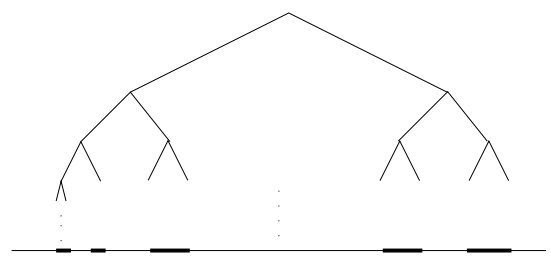

Fig. 2

Draw in the plane the complete binary tree using straight line segments so that the infinite branches of the tree converge to all points of a Cantor set $P$ which is contained in the closed line segment $[0,1]$. Let $S$ be the tree together with $[0,1]$.

The set $\operatorname{lnc}(S)$ consists of all points of $P$ together with all branch-points of the tree. The set of lnc points of that set is $P$; and now the derivation process stabilizes, with a perfect set remaining. However, $S$ is countably convex, so $B(S)=\emptyset$.

Since $\gamma(S)$ is equal to the chromatic number of $H(S)$ it is reasonable to look for a definition of a derived set which uses both topology and the structure of $H(S)$. This is done next. The transfinite process in the definition below does indeed exhaust all points of $A(S)$, leaving $B(S)$ as the remaining perfect set - when it is applied to closed sets.

Definition 7. Let $S$ be a subset of a topological vector space. By induction on ordinals $\alpha$ we define when $\varrho(x) \geq \alpha$ holds for $x \in S$. 
(i) $\varrho(x) \geq 0$ for all $x \in S$.

(ii) If $\alpha$ is a limit ordinal then $\varrho(x) \geq \alpha$ iff $\varrho(x) \geq \beta$ for every $\beta<\alpha$.

(iii) $\varrho(x) \geq \alpha+1$ iff for every open $u \ni x$ there exists a finite $X \subseteq S \cap u$ so that $\varrho(y) \geq \alpha$ for all $y \in X$ and $X$ is defected in $S$.

Let

$$
\varrho(x)= \begin{cases}\min \{\alpha: \varrho(X) \nsupseteq \alpha+1\} & \text { if such an } \alpha \text { exists, } \\ \infty & \text { otherwise. }\end{cases}
$$

Put, formally, $\alpha<\infty$ iff $\alpha$ is an ordinal.

Definition 8. For every subset $S$ of a topological vector space let $\varrho(S)=$ $\sup \{\varrho(x): x \in S\}$.

The following follows directly from the definition of $\varrho$ :

FACT 9. (i) If $\varrho(x) \geq \alpha$ then $\varrho(x) \geq \beta$ for all $\beta<\alpha$.

(ii) The set $\{\varrho(x) \geq \theta\}$ is closed in $S$ for every $\theta \in \mathrm{On} \cup\{\infty\}$.

Let us also remark that in a normed space a point has rank $\geq \alpha+1$ in a set $S$ if and only if it is a limit of a sequence $\left(x_{n}\right)_{n}$ of points of rank $\geq \alpha$ with the property that for every $n$ there is a finite set $X \subseteq \mathbb{N}-\{0,1, \ldots, n\}$ so that $\left\{x_{m}: m \in X\right\}$ is defected in $S$.

In the set $S$ in Figure 3, the points of rank 0 are the lc points, the points of rank 1 are the branch-points in the tree and the points of rank 3 are all points of $P$. There are no points of rank $\geq 4$ since there is no finite defected subset of $P$, as $P$ is contained in a convex subset of $S$.

LEMma 10. Suppose that $S$ is a subset of a second countable topological vector space. Let $\alpha$ range over ordinal numbers.

(i) If $\varrho(x)=\alpha$ then there is some open $u \ni x$ so that if $X \subseteq u$ is finite and $\varrho(y)=\alpha$ for all $y \in X$ then $\operatorname{conv}(X) \subseteq S$.

(ii) If $\varrho(x)=\alpha$ then there is some open $u \ni x$ so that $\operatorname{conv}\{y \in u$ : $\varrho(y)=\alpha\} \subseteq S$.

(iii) If $\varrho(x)=\alpha$ then there is an open $u \ni x$ so that $\varrho(y) \leq \alpha$ for all $y \in u$.

(iv) If $\varrho(x)=\alpha$ then for every open $u \ni x$ and $\beta<\alpha$ there is $y \in u$ so that $\varrho(y)=\beta$.

(v) There exists some countable ordinal $\alpha$ ( $a$ "threshold" ordinal) so that $\varrho(x)<\infty \Rightarrow \varrho(x)<\alpha$.

(vi) All points $x$ with $\varrho(x)<\infty$ are covered by countably many convex subsets of $S$.

(vii) The set $K=\{x: \varrho(x)=\infty\}$ is closed in $S$ and for every $x \in K$ and open $u \ni x$ there is a finite defected $X \subseteq u \cap K$.

Proof. (i) If in every open $u \ni x$ there is a defected $X$ with $\varrho(y)=a$ for all $y \in X$ then $\varrho(x) \geq \alpha+1$, contrary to $\varrho(x)=\alpha$. 
(ii) Follows from (i). Fix $u \ni x$ so that every finite $X \subseteq u \cap\{y: \varrho(y)=\alpha\}$ is nondefected, that is, $\operatorname{conv}(X) \subseteq S$. Since $\operatorname{conv}\{y \in u: \varrho(y)=\alpha\}$ is the union of the convex hulls of its finite subsets, (ii) follows.

(iii) A consequence of the fact that $\{x: \varrho(x) \geq \alpha+1\}$ is closed in $S$.

(iv) By induction on $\alpha$. If $\alpha=0$ there is nothing to prove.

Suppose $\varrho(x)=\alpha>0$ and (iv) holds for all $\gamma<\alpha$. Let $\beta<\alpha$ and an open $u \ni x$ be given. By shrinking $u$ it may be assumed that $\varrho(y) \leq \alpha$ for all $y \in u$, by (ii), and that if $X \subseteq u$ is defected then there is some $y \in X$ so that $\varrho(y)<\alpha$, by (i). Since $\varrho(x) \geq \beta+1$ there is a finite defected $X \subseteq u$ so that $\varrho(y) \geq \beta$ for all $y \in X$. Pick $y \in X$ so that $\varrho(y)<\alpha$. If $\varrho(y)=\beta$ then the proof is done. Otherwise, $\varrho(x)=\gamma$ where $\beta<\gamma<\alpha$ and the induction hypothesis for $\gamma$ is used to find $y \in u$ so that $\varrho(y)=\beta$.

(v) Suppose (v) fails. Then for every $\alpha<\omega_{1}$ there exists $x \in S$ with $\alpha<\varrho(x)<\infty$. Therefore, by (iv), for every $\alpha<\omega_{1}$ some $x_{\alpha} \in S$ can be fixed with $\varrho\left(x_{\alpha}\right)=\alpha$. Let $\mathcal{B}$ be a countable base for the topology. For every $\alpha<\omega_{1}$ there exists some $u \in B$ that separates $x_{\alpha}$ from $\left\{x_{\beta}: \alpha<\beta<\omega_{1}\right\}$ by (ii), which is impossible, as $\mathcal{B}$ is countable.

(vi) Fix a countable base $\mathcal{B}$ for the topology. Let $\alpha<\omega_{1}$ be the threshold ordinal from (v). For every $\beta<\alpha$ and open $u \in \mathcal{B}$ let $c_{\beta, u}:=\operatorname{conv}\{y \in u$ : $\varrho(y)=\beta\}$. The set $\{x: \varrho(x)=\beta\}$ is covered by $\left\{c_{u, \beta}: u \in \mathcal{B}\right.$ and $\left.c_{u, \beta} \subseteq S\right\}$ by (iii).

(vii) Fix some $x$ with $\varrho(x)=\infty$ and let $\alpha$ be the threshold ordinal from (v). Let $u \ni x$ be an arbitrary open neighborhood. Since $\varrho(x) \geq \alpha+1$ there exists a finite defected $X \subseteq u$ so that $\varrho(y) \geq \alpha$ for all $y \in u$. But $\varrho(y) \geq \alpha$ implies that $\varrho(y)=\infty$, hence $\varrho(y)=\infty$ for all $y \in X$.

REMARK. The lemma holds for topological vector spaces which are not second countable, with obvious modifications. If the weight of the space is $\kappa$, then the threshold ordinal is below $\kappa^{+}$, the successor cardinal of $\kappa$, and all points of $\varrho$-degree below $\kappa^{+}$are coverable by $\kappa$ convex subsets of $S$.

Definition 11. For every set $S$ in a topological vector space let the convexity radical of $S, K(S)$, be defined by

$$
K(S)=\{x \in S: \varrho(x)=\infty\} .
$$

The radical $K(S)$ has a direct, equivalent definition.

Definition 12. (i) A semi-clique in $S$ is a subset $Q \subseteq S$ so that for every $x \in Q$ and open $u \ni x$ there exists a finite subset of $Q \cap u$ which is defected in $S$.

(ii) The union of all semi-cliques in $S$ is a closed, maximal semi-clique in $S$, which is denoted by $C(S)$.

FACT 13. For every subset $S$ of a topological vector space, $C(S)=K(S)$. 
Proof. For every set $S, K(S)$ is a semi-clique by Lemma 10 (vii) and therefore $K(S) \subseteq C(S)$.

Conversely, by induction on $\alpha$ it is seen that $\varrho(x) \geq \alpha$ for all $x \in C(S)$. If $\alpha$ is limit then $\varrho(x) \geq \alpha$ for $x \in S$ by the induction hypothesis and if $\varrho(x) \geq \alpha$ for all $x \in C(S)$ then $\varrho(x) \geq \alpha+1$ for all $x \in C(S)$ by the definition of semi-clique. Therefore $C(S) \subseteq K(S)$.

FACT 14. If $S$ is a subset of a second countable topological vector space then $B(S) \subseteq K(S)$.

Proof. By Fact 6(iii), $B(S)$ is a semi-clique in $S$, and therefore $B(S) \subseteq$ $C(S)=K(S)$.

By this fact, for every $S$ in a second countable topological vector space, $\left\{x \in S: \varrho(x)<\omega_{1}\right\} \subseteq A(S)$. The proof of Lemma 10(vi) gives an "effective" inductive procedure for covering the former set by countably many convex subsets of $S$. If $B(S)=K(S)$ then this procedure actually exhausts $A(S)$, the countably convex part of $S$. Unfortunately, it can happen that $B(S) \varsubsetneqq$ $K(S)$ :

EXAMPLE 15. Let

$$
L_{r}=\{(r, y): y \in \mathbb{R}\}, \quad S=\bigcup_{r \in \mathbb{Q}} L_{r} .
$$

The set $S$ is countably convex, so $B(S)=\emptyset$, but $C(S)=K(S)=S$. In other words, the set $K(S)$ may be strictly bigger than $B$, even in descriptively simple Borel sets - the set in the example is $F_{\sigma}$.

An example of a countably convex $G_{\delta}$ set in $\mathbb{R}^{2}$ with nonempty convexity radical will be presented in [7], which is devoted to $G_{\delta}$ sets. Open sets in Polish spaces are of course countably convex, so the only class of sets in the Borel hierarchy for which there is still hope that the radical always equals $B$ is the class of closed sets.

Theorem 16. If $S$ is a closed subset of a Polish vector space, then $B(S)=K(S)$.

Pr o of. Suppose that $x \in K$ and that $u \ni x$ is open. If $K \cap u \subseteq \bigcup_{i \in \mathbb{N}} D_{i}$, where $D_{i} \subseteq S$ for all $i$, then, since $K$ is a closed subset of $S$, which is closed in a Polish space, there exists $i$, say $i=0$, so that $D_{i} \cap K \cap v$ is dense in $v \cap K$ for some open $v \subseteq u$. If $D_{0}$ is a convex subset of $S$, then so is its closure. But then $C \cap v \subseteq \operatorname{cl} D_{0}$ and contains defected sets - a contradiction. Therefore $D_{0}$ is not convex and consequently $\gamma(S \cap u)>\aleph_{0}$.

Corollary 17. A closed set in a Polish linear space is a countable union of convex sets if and only if $\varrho(S)<\omega_{1}$. 
Proof. If $\varrho(S)<\omega_{1}$ then $K(S)=\emptyset=B(S)$ and therefore $\gamma(S) \leq \aleph_{0}$. Conversely, if $K(S)=B(S) \neq \emptyset$ then $\gamma(S)>\aleph_{0}$ by Fact 6 (iv).

Corollary 17 and Theorem 16 divide closed countable unions of convex sets in Polish linear spaces into $\aleph_{1}$ different types by their $\varrho$ rank.

The definition of $\varrho$ makes it clear that $x \in \operatorname{lnc} S$ if and only if $\varrho(S) \geq 1$. The function $\varrho$ ranks, then, the complexity of local nonconvexity points, and highlights a qualitative difference between points whose rank is countable and points in the convexity radical, whose complexity is "unbounded". The theorems in this section can be understood as stating the equivalence of countable convexity with the absence of "unboundedly complicated" lnc points.

A closed, connected subset of a topological vector space which has no lnc points is convex by a theorem of Tietze (see [8]), so Theorem 16 can be regarded as a countable generalization of Tietze's theorem.

4. Unit spheres in separable Banach spaces. Let $X$ be a separable Banach space, and let $S$ be the unit sphere of $X$. By the previous section, $S$ is countably convex if and only if $\varrho(S)<\omega_{1}$. Can a sphere in a separable Banach space be countably convex? In $\mathbb{R}^{n}$ with the usual Euclidean norm, the only convex subsets of the unit sphere are the singletons, so $\varrho(S)=\infty$, but under the maximum norm the unit sphere in $\mathbb{R}^{n}$ is a polytope, and $\varrho(S)=n+1$.

Consider now the class of separable Banach spaces $C(K), K$ a compact metric space, with the natural sup-norm and with other, equivalent, norms.

Suppose $K$ is an uncountable compact metric space. For every $x \in K$ there is a continuous function $f_{x}$ on $K$ with $f(x)=1$ and $|f(y)|<1$ for all $x \neq y \in K$. Since the convex hull of $\left\{f_{x}, f_{y}\right\}$ is not contained in the unit sphere for any two distinct $x, y \in K$, the set $\left\{f_{x}: x \in K\right\}$ is an uncountable visually independent subset of the unit sphere of $C(K)$ with the natural norm - which forces that the sphere is not countably convex.

Moreover, the unit sphere in $C(K)$ for uncountable $K$ is not countably convex with respect to any norm which is equivalent to the natural one. There are two ways to see this: the space $C(\Delta)$ of all continuous functions on the Cantor set with the natural norm is isometrically universal for separable Banach spaces by the Banach-Mazur theorem, and therefore contains isometrically some reflexive Banach space. Milyutin's theorem [18] asserts that $C(K)$ and $C(\Delta)$ are isomorphic, so $C(K)$ with any equivalent norm contains an isomorphic copy of a reflexive Banach space, which is itself reflexive. By [16], the unit sphere of a reflexive Banach space is never countably convex, so neither is the unit sphere of $C(K)$.

Alternatively, $C(\Delta)$ is isometrically embedded in $C(K)$ with the natural norm by [15], hence contains an isometric copy of a reflexive Banach space. 
If we change the natural norm on $C(K)$ to an equivalent one, then this copy remains reflexive, and hence the unit sphere in $C(K)$ cannot be countably convex.

Let $K$ be a countable compact metric space. There exists a strictly convex norm which is equivalent to the sup-norm in $C(K)$ (see [5]), of which the sphere has no convex subsets except singletons. But the sphere of $C(K)$ with the sup-norm is indeed countably convex, and its rank will be calculated next.

4.1. The rank of the unit sphere of $C(K)$ for countable compact $K$. A formula for the convexity rank of the sphere in $C(K)$ for countable metric compact $K$ will now be given in terms of the Cantor-Bendixson degree of $K$.

Let $K$ be a countable compact Hausdorff space. For every subset $A \subseteq K$ the derived set $A^{\prime}$ of $A$ is the set of all accumulation points of $K$. The $\alpha$ th derived set of $A, A^{(\alpha)}$, is defined by induction on ordinals $\alpha$ by $A^{(\alpha+1)}=$ $\left(A^{(\alpha)}\right)^{\prime}$ and $A^{(\alpha)}=\bigcap_{\beta_{\alpha}} A^{(\beta)}$ for limit $\alpha$. Since every closed set in a countable compact Hausdorff space contains isolated points, $A^{\prime} \mp A$ for every closed $A \subseteq K$ and therefore there is some ordinal $\alpha<\omega_{1}$ so that $K^{(\alpha)}=\emptyset$. The first $\alpha=\alpha(K)$ for which $K^{(\alpha)}$ is finite is the Cantor-Bendixson degree of $K$. Define $\left|K^{(\alpha)}\right|=n(K)<\omega$. By compactness, $n(K)>0$.

Countable successor ordinals $\gamma$ with the order topology constitute all examples, up to homeomorphism, of countable compact metric spaces. An ordinal $\gamma$ is compact with the order topology if and only if it is a successor ordinal.

Recall that ordinal multiplication is defined by $\alpha 0=0, \alpha(\beta+1)=$ $\alpha \beta+\alpha$ and $\alpha \beta=\lim _{\gamma<\beta} \alpha \gamma$ for limit $\beta$; ordinal exponentiation is defined analogously. For every ordinal $\gamma$ there is a unique way to write $\gamma$ in base $\omega$, that is, as $\gamma=\sum_{i<l} \omega^{\alpha_{i}} n_{i}$ where $\alpha_{i}>\alpha_{i+1}$. In particular, $\gamma$ can be written uniquely as $\omega^{\alpha} n+\delta$ where $\delta<\omega^{\alpha}$.

A countable compact Hausdorff $K$ with $\alpha(K)=\alpha$ and $n(K)=n$ is homeomorphic to $\omega^{\alpha} n+1$ with the order topology, and thus the pair $(\alpha(K), n(K))$ is a complete homeomorphism invariant of countable metric compact $K$. In particular, $\gamma, \beta<\omega_{1}$ are homeomorphic as compact metric spaces iff $\alpha_{1}=\alpha_{2}$ whenever $\gamma=\omega^{\alpha_{1}}+\delta_{1}, \beta=\omega^{\alpha_{2}}+\delta_{2}$ with $\delta_{i}<\omega^{\alpha_{i}}$, $i=1,2$.

Every countable compact $K$ is homeomorphic to a well ordered subset of the rationals with the induced metric. It is convenient to identify such a $K$ with a well ordered subset of $\mathbb{Q}$.

TheOREM 18. Suppose $K$ is a countable compact metric space. Let $\alpha(K)=\alpha, n(K)=n$, and let $S$ be the unit sphere of $C(K)$ with the sup-norm. Then

$$
\varrho(S)=\omega \alpha+n-1 .
$$


Pr o o f. For every $f \in S$ let $M(f)=\{x \in K:|f(x)|=1\}$. Then $M(f)$ is closed and nonempty for each $f \in S$. Let $\alpha(f)$ be the maximal $\alpha$ for which $M(f) \cap K^{(\alpha)}$ is nonempty. Then $M(f) \cap K^{(\alpha(f))}$ is necessarily finite. Define $n(f)=|M(f) \cap \alpha(f)|$. Let $\operatorname{tp}(f)=(\alpha(f), n(f))$. If $f \in S$ is constant, then $(\alpha(f), n(f))=(\alpha(K), n(K))$.

The theorem follows by applying the following lemma to one of the two constant functions in $S$.

Lemma 19. For every countable compact Hausdorff space $K$, and $f$ in the unit sphere of $C(K)$ with the natural norm,

$$
\varrho_{S}(f)=\omega \alpha(f)+n(f)-1 .
$$

Let $K$ be a well ordered subset of $\mathbb{Q}$.

First, it is proved by induction on $\alpha(f) \omega+n(f)$ that

$$
\varrho(f) \geq \omega \alpha(f)+n(f)-1 .
$$

FiRst CASE: $n=1$. Suppose that $f \in S$ and $\operatorname{tp}(f)=(\alpha, 1)$. If $\alpha=0$ then (4) states that $\varrho(f) \geq 0$, which is trivially true. Suppose, then, that $\alpha>0$ and let $q$ be the unique point in $K^{(\alpha)}$ for which $|f(q)|=1$. To prove (4) it suffices to show that for every given $\varepsilon>0, \beta<\alpha$ and $m \in \mathbb{N}$ there exists a function $g \in S$ with $\operatorname{tp}(g)=(\beta, m)$ and $\|g-f\|<\varepsilon$.

Suppose $1>\varepsilon>0$ is given. Let $r<q$ be such that $(r, q) \cap K^{(\alpha)}=\emptyset$ and $|f(x)-f(q)|<\varepsilon$ for all $x \in(r, q]$. Let $t_{1}, \ldots, t_{m} \in(r, q)$ be points in $K^{(\beta)}$ and let $r_{i}<t_{i}$ be so chosen that $r_{i}$ is irrational, $\left(r_{i}, t_{i}\right] \subseteq(r, q)$ and $\left(r_{i}, t_{i}\right) \cap K^{(\beta)}=\emptyset$. Since each interval $u_{i}=\left(r_{i}, t_{i}\right]$ is clopen in $K$, the following function $g$ is continuous:

$$
g(x)= \begin{cases}f(q) & \text { if } x \in \bigcup_{i=1}^{n}\left(r_{i}, t_{i}\right], \\ (1-\varepsilon) f(x) & \text { otherwise. }\end{cases}
$$

Also, $\|g-f\| \leq \varepsilon$ and $g \in S$. Finally, $\operatorname{tp}(g)=(\beta, m)$ since $t_{1}, \ldots, t_{m}$ are the only points from $K^{(\beta)}$ at which $|g|=1$, and $|g(x)|<1$ for all $x \in K^{(\alpha)}$.

SECOND CASE: $n>1$. Let $q_{0}, \ldots, q_{n-1}$ be all points of $M(f) \cap K^{(\alpha)}$. Let $1>\varepsilon>0$ be given.

Find, for each $i<n$, an irrational $r_{i}<q_{i}$ so that $\left|f(x)-f\left(q_{i}\right)\right|<\varepsilon$ for each $x \in\left(r_{i}, q_{i}\right)$ and $\left(r_{i}, q_{i}\right) \cap K^{(\alpha)}=\emptyset$. Define $g_{i}$ by

$$
g_{i}(x)= \begin{cases}f(x) & \text { if } x \in \bigcup_{j \neq i}\left(r_{j}, q_{j}\right], \\ (1-\varepsilon) f(x) & \text { otherwise. }\end{cases}
$$

Since for each $i<n, K \cap\left(r_{i}, q_{i}\right]$ is clopen in $K, g_{i}$ is continuous. Since $n>1, g_{i} \in S$. Also, $\left\|g_{i}-f\right\| \leq \varepsilon$. Finally, $n^{-1} \sum_{i<n} g_{i} \notin S$. Since $\operatorname{tp}\left(g_{i}\right)=$ $(\alpha, n-1)$, the induction hypothesis gives $\varrho\left(g_{i}\right) \geq \alpha \omega+n-2$, and this establishes that $\varrho(f) \geq \omega \alpha+n-1$. 
Now we prove

$$
\varrho(f) \leq \omega \alpha(f)+n(f)-1 .
$$

Suppose to the contrary that $\operatorname{tp}(f)=(\alpha, n)$ and $\varrho(f) \geq \omega \alpha+n$. Let $\left\{q_{0}, \ldots, q_{n-1}\right\}=M(f) \cap K^{(\alpha)}$ and $\varepsilon=\min \left\{1-|f(x)|: x \in K^{(\alpha)}-\right.$ $\left.\left\{q_{0}, \ldots, q_{n-1}\right\}\right\}$. Since $K^{(\alpha)}$ is compact and each $q_{i}$ is isolated in $K^{(\alpha)}$, it follows that $\varepsilon>0$. Since $\varrho(f) \geq \omega \alpha+n$, there exists a set $\left\{g_{j}: j<l\right\}$ $\subseteq S \cap B(f, \varepsilon)$ so that $\varrho\left(g_{j}\right) \geq \alpha \omega+n-1$ for each $j<l$, and a convex combination $\sum_{j<l} a_{j} g_{j}$ is not in $S$. By the induction hypothesis $\operatorname{tp}\left(g_{j}\right) \geq$ $(\alpha, n)$ for each $j<l$. Since $\left|M\left(g_{j}\right) \cap K^{(\alpha)}\right| \geq n$, but $\left|g_{j}(x)\right|<1$ for each $x \in K^{(\alpha)}-\left\{q_{0}, \ldots, q_{n-1}\right\}$, necessarily $g_{j}\left(q_{i}\right)=f\left(q_{i}\right)$ for all $i<n$. But then also $\sum_{j<l} a_{j} g_{j}\left(q_{i}\right)=f\left(q_{i}\right)$ for each $i<n$, contrary to $\sum_{j<l} a_{j} g_{j} \notin S$.

COROllary 20. For every countable ordinal $\beta$ there exists a unique compact metric space $K$ so that $\varrho\left(S_{K}\right)=\beta$.

Pro of. Given $\beta<\omega_{1}, \beta$ can be written uniquely as $\omega \alpha+n$ for some $\alpha<$ $\omega_{1}$ and natural $n \geq 0$. The unique compact metric $K$ with $(\alpha(K), n(K))=$ $(\alpha, n)$ satisfies $\varrho\left(S_{K}\right)=\beta$ by formula $(3)$.

Thus, the convexity type $(\alpha, n)$ of the unit sphere in $C(K)$ with the natural norm is another complete homeomorphism invariant of countable compact metric spaces.

For every countable compact ordinal $\gamma+1$ with the order topology,

$$
\alpha(\gamma+1) \leq \varrho(\gamma+1) \leq \gamma .
$$

For example, for $K=\omega+1, C(K)$ is $c$, the space of all convergent real sequences, $\alpha(K)=1, \varrho(K)=\omega$; for $K=\omega^{2}+1, \alpha(K)=2$ and $\varrho(K)=\omega 2$.

Still, for uncountably many countable ordinals $\gamma$-all those $\gamma$ which satisfy $\gamma=\omega^{\gamma}$-we have $\alpha(\gamma+1)=\varrho(g+1)=\gamma+1$.

5. Uncountable convexity. Remove from the plane a countable dense subset. The remaining set $S$ is a $G_{\delta}$ which satisfies $K(S)=S$ and $\gamma(S)>$ $\aleph_{0}$. Now consider all the circles with center at the origin. Since there are uncountably many disjoint circles like that, there exists one such circle which is entirely contained in $S$. The circle is a perfect subset of $S$ with the property that all of its 3 -element subsets are defected in $S$ - that is, the circle is a perfect 3 -clique in $S$.

Once a set contains a 3 -clique of cardinality $2^{\aleph_{0}}$, its convexity number has to be continuum, since any convex subset of the set can contain at most two elements from the clique. Therefore, it is impossible to find a $G_{\delta}$ set in $\mathbb{R}^{2}$ whose convexity number consistently violates the continuum hypothesis.

There is a crucial difference between the behavior of uncountably convex sets in $\mathbb{R}^{2}$ to their behavior in higher dimensions. In higher dimensions a 
closed set can fail to be countably convex without containing uncountable cliques. Such a set is constructed next.

The difference between $\mathbb{R}^{2}$ and $\mathbb{R}^{3}$ runs, however, even deeper than that, since the convexity number of the closed set constructed here consistently violates the continuum hypothesis.

TheOREM 21. There exists a compact, simply connected $S \subseteq \mathbb{R}^{3}$ so that $\varrho(S)=\infty$ and all cliques in $S$ are countable; in fact, the set of accumulation points of each clique in $S$ is finite.

Proof. Let $D$ be the closed unit ball in $\mathbb{R}^{3}$. A closed, simply connected $S \subseteq D$ is constructed by removing from $D$ infinitely many pairwise disjoint open pyramidal indentations. The removal is performed by induction on $n$, and the indentations are indexed by finite sequences over the symbols $\{0,1,2\}$. Each indentation $T_{\eta}$ for a sequence $\eta$ is contained in the convex hull of a closed spherical cap $c_{\eta}$. Let $3^{n}$ denote the collection of all sequences of length $n$ over $\{0,1,2\}$. Denote by $3^{\leq n}$ all sequences over $\{0,1,2\}$ of length at most $n$ and similarly for $3^{<n}$. The empty sequence is denoted by \langle\rangle .

Let $c_{\langle\rangle}$be some hemisphere in $D$.

Suppose that $c_{\eta}$ is defined for all $\eta \in 3^{n}$ and that $T_{\nu}$ is defined for all $\nu \in 3^{<n}$. Here is how $T_{\eta}$ and $c_{\eta \uparrow i}$ for $i=0,1,2$ are defined.

Call a set $\{a, b, c\} \subseteq \mathbb{R}^{3}$ almost equilateral if the ratio between any two distances in the set does not exceed 1.1.

Let $a_{0}^{\prime}, a_{1}^{\prime}, a_{2}^{\prime}$ belong to the relative boundary of $c_{\eta}$ and form the vertices of an equilateral triangle. Let $o^{\prime}$ denote the center of $c_{\eta}$. Let $a_{i}$ be the middle point of the geodesic $\left(a_{i}^{\prime}, o^{\prime}\right)$. Let $P_{i}$ be the plane through $\left(a_{i}^{\prime}, a_{j}, a_{k}\right)$ (where $\{i, j, k\}=\{0,1,2\}$ ) and let $\bar{P}_{i}$ be the open half-space supported by $P_{i}$ which does not contain the origin. Now define $T_{\eta}=D \cap \bigcap_{i=0,1,2} \bar{P}_{i}$. The intersection $\bar{P}_{i} \cap D$ is contained in the convex hull of $c_{\eta}$, and therefore $P_{i}$ separates $T_{\eta}$ from $D-\operatorname{conv}\left(c_{\eta}\right)$, and in particular,

$$
T_{\eta} \subseteq \operatorname{conv}\left(c_{\eta}\right) .
$$

To define $c_{\eta \uparrow i}$ for $i<3$, let $d_{i}$ be on the geodesic $\left(a_{i}, a_{i}^{\prime}\right)$ at distance $\delta$ from $a_{i}$. The distance $\delta$ should be so small that the plane spanned by $\left(d_{0}, d_{1}, d_{2}\right)$ meets $T_{\eta}$. Let $c_{\eta \uparrow}$ be a closed spherical cap with center $d_{i}$ and radius $r$. The radius is chosen to be sufficiently small so that each $c_{\eta \uparrow} i$, for $i<3$, avoids $a_{i}$ and for every choice of $x_{i} \in c_{\eta^{\wedge} i}$,

$$
\begin{aligned}
& \left\{x_{0}, x_{1}, x_{2}\right\} \text { is almost equilateral, } \\
& \operatorname{conv}\left(x_{0}, x_{1}, x_{2}\right) \text { meets } T_{\eta} .
\end{aligned}
$$

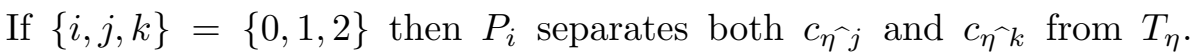
Therefore, since $P_{i}$ also separates $D-\operatorname{conv}\left(c_{\eta}\right)$ from $T_{\eta}$, the following is true: 
FACT 22. If $\{i, j\} \subseteq\{0,1,2\}$ then for every subset $Y \subseteq D-\operatorname{conv}\left(c_{\eta}\right)$ we have $\operatorname{conv}\left(c_{\eta^{\wedge} i} \cup c_{\eta^{\wedge} j} \cup Y\right) \cap T_{\eta}=0$.

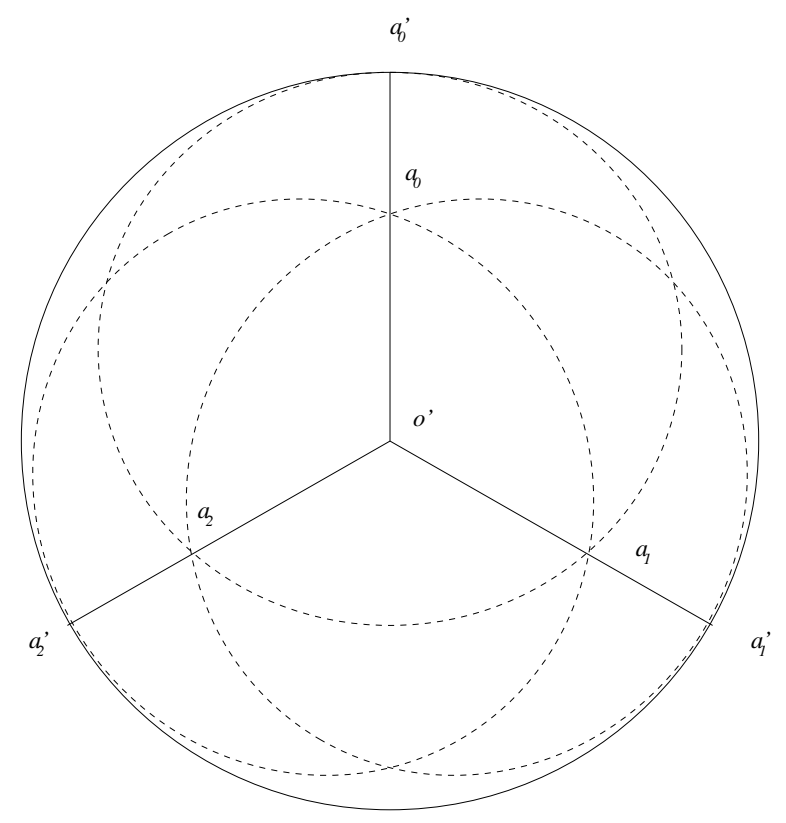

Fig. 3

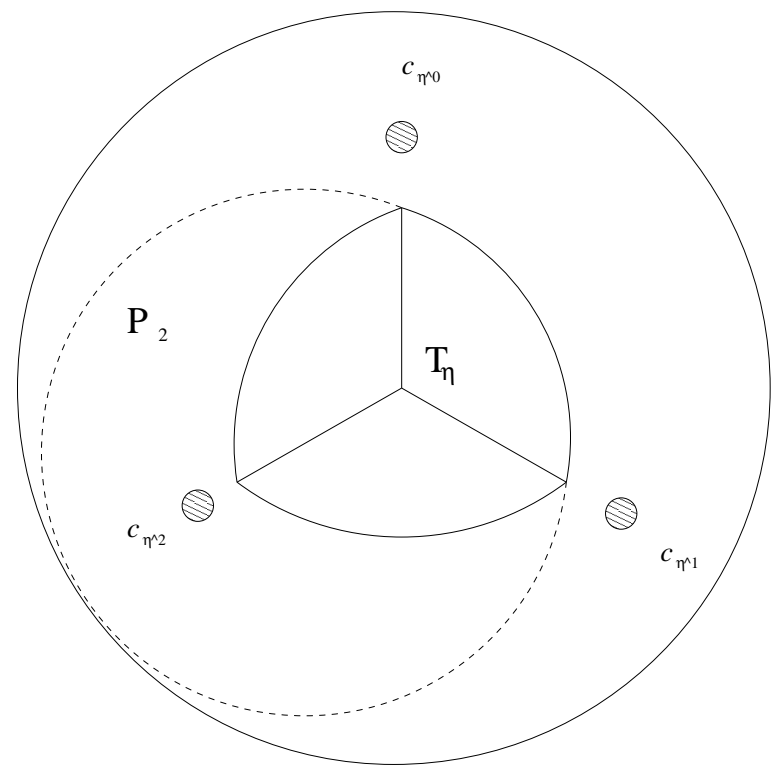

Fig. 4 
In particular, $\operatorname{conv}\left(c_{\eta \uparrow i}\right) \cap T_{\eta}=\emptyset$ for all $i<3$. Also, $P_{i}$ separates $\operatorname{conv}\left(T_{\eta_{\uparrow} i}\right)$ and therefore also $T_{\eta^{\wedge} i}$ from each of $c_{\eta^{\wedge} j}, c_{\eta \uparrow k}$, which contain, respectively, $T_{\eta^{\wedge} j}$ and $T_{\eta^{\wedge} k}$. Therefore for any two finite sequences $\eta, \nu$,

$$
\eta \neq \nu \Rightarrow T_{\eta} \cap T_{\nu}=\emptyset .
$$

Let $S_{n}=D-\bigcup_{\eta \in 3 \leq n} T_{\eta}$. Let $S=\bigcap_{n} S_{n}$. The set $S$ is compact and simply connected.

Let $K=\bigcap_{n} \bigcup_{\eta \in 3^{n}} c_{\eta}$. More specifically, for an infinite sequence $\eta$ over $0,1,2$ let $x_{\eta}=\bigcap_{n} c_{\eta \mid n}$. The correspondence between $\eta$ and $x_{\eta}$ is a 1-1 correspondence between elements of $K$ and infinite sequences over $\{0,1,2\}$.

Lemma 23. A 3-element subset of $K$ is defected in $S$ if and only if it is almost equilateral.

P r o o f. Let $x, y, z$ be three distinct elements of $K$. If there exists a finite sequence $\eta$ over $\{0,1,2\}$ so that $\{x, y, z\}$ meets each $c_{\eta \uparrow i}$ for $i<3$, then $\{x, y, z\}$ is both almost equilateral and defected in $S$ by (8) and (9) above.

Otherwise, for every $\eta,\{x, y, z\}$ meets at most two of $c_{\eta i}$ for $i<3$. Let $n$ be the largest so that there exists some $\eta \in 3^{n}$ with $\{x, y, z\} \subseteq c_{\eta}$. On the one hand, $\{x, y, z\} \subseteq \bigcup_{i<3} c_{\eta \uparrow}$, and on the other hand, there must be some $i<3$, say $i=0$, so that $\{x, y, z\} \cap c_{\eta \wedge}=\emptyset$. Since not all of $\{x, y, z\}$ belong to neither $c_{\eta \uparrow 1}$ nor $c_{\eta\urcorner 2}$ by the choice of $\eta$, necessarily two of the points are in, say, $c_{\eta \uparrow 2}$ and the third is in $c_{\eta \uparrow 1}$. Hence $\{x, y, z\}$ is not almost equilateral.

To see that $\{x, y, z\}$ is not defected consider an arbitrary sequence $\eta$. Since $\{x, y, z\}$ meets at most two of $c_{\eta \wedge}$, assume $\{x, y, z\} \cap c_{\eta \wedge 2}=\emptyset$. Then $\{x, y, z\} \subseteq c_{\eta\urcorner 0} \cup c_{\eta\urcorner 1} \cup D-\operatorname{conv}\left(c_{\eta}\right)$ and therefore $\operatorname{conv}(x, y, z) \cap T_{\eta}$ $=\emptyset$ by Fact 22 .

Since $\eta$ is arbitrary, $\operatorname{conv}(x, y, z)$ does not meet any $T_{\eta}$ and thus $\operatorname{conv}(x, y, z) \subseteq S$.

Lemma $24 . K$ is a semi-clique in $S$.

Proof. Let $x=x_{\eta}$ be an arbitrary member of $K$ and let $u \ni x_{\eta}$ be open. Let $n$ be so large that $c_{\eta \uparrow n} \subseteq u$. Let $\nu_{0}, \nu_{1}, \nu_{2}$ be three infinite sequences with $\nu_{i} \uparrow(n+1)=\left(\eta\lceil n) \uparrow i\right.$. The corresponding points $x_{\nu_{i}}$ belong to $K$ and their convex hull meets $T_{\eta\lceil n}$ (since $x_{\nu_{i}} \in c_{\left(\eta\lceil n)^{\wedge} i\right.}$ ). Therefore $\left\{x_{\nu_{0}}, x_{\nu_{1}}, x_{\nu_{2}}\right\}$ is a finite defected subset of $P \cap u$.

Corollary 25. $\gamma(S)>\aleph_{0}$.

Lemma 26. Every $x \in S-K$ satisfies $\varrho(x) \leq 1$; hence $K$ is the convexity radical of $S$.

Proof. If $x$ belongs to the interior of $S$ then $\varrho(x)=0$, so assume that $x \in \operatorname{bd} S$. If $x$ does not belong to bd $D$ then it necessarily belongs to the 
boundary of some $T_{\eta}$, in which case it has either rank 1 if it belongs to a side of $T_{\eta}$ or else has rank 0 .

Assume then that $x \in \mathrm{bd} S \cap \mathrm{bd} D$. Since $x \notin K$ there is some $n$ so that $x \notin \bigcup_{\eta \in 3^{n}} c_{\eta}$. Fix an open $u \ni x$ so that $u \cap \bigcup_{\eta \in 3^{n}} \operatorname{conv}\left(c_{\eta}\right)=\emptyset$. Since $S \cap u=S_{n} \cap u$ it follows that $\varrho(x)=0$.

Lemma 27. If $Q \subseteq S$ is a clique then the set of accumulation points of $Q$ is finite.

Proof. First observe that if $x \in A$ is an accumulation point of some clique $Q \subseteq S$ then necessarily $x \in K$, because for every point $x \in S-K$ there exists an open $u \ni x$ so that $S \cap u$ is the union of at most 3 free sets in $H(S)$.

Suppose to the contrary that $Q \subseteq S$ is a clique and $Q_{0} \subseteq Q$ is an infinite set of accumulation points of $Q$. By the previous observation, $Q_{0} \subseteq K$. As $S$ is compact, there exists $x \in S$ which is an accumulation point of $Q_{0}$. Define by induction a sequence $\left(x_{n}\right) \subseteq Q_{0}$. Let $x_{0} \neq x$ be chosen in $Q_{0}$. Suppose $x_{n}$ is defined. Let $x_{n+1} \neq x$ be chosen in $B(x, \varepsilon) \cap Q_{0}$ where $\varepsilon \leq d\left(x_{n}, x\right) / 10$.

In $\left\{x_{n}\right\}$ there is no almost equilateral triangle. Since $\left\{x_{n}\right\} \subseteq K$, by Fact 23, $\left\{x_{n}\right\}$ contains no defected triples. Since $S$ is simply connected, $\left\{x_{n}\right\}$ contains no defected subsets at all-contrary to $\left\{x_{n}\right\} \subseteq Q$.

REMARK. For every $n, S$ contains cliques with $n$ accumulation points. To see this choose $n$ points from $K(S)$ and find for each point a visually independent sequence which converges to the point. The union of those sequences is an $n+1$-clique.

Corollary 28. Every clique in $S$ is countable.

P r o o f. Since every uncountable subset of $\mathbb{R}^{3}$ contains uncountably many limits of itself, every clique in $S$ is countable by Lemma 27 .

This completes the proof of Theorem 21 .

6. Independence of $\gamma(S)$. Suppose $S$ is a subset of a linear space. Let $\mathcal{I}$ be the $\sigma$-ideal generated by all convex subsets of $S$. This ideal is proper if and only if $\gamma(S)>\aleph_{0}$, and when it is proper, there are several standard set-theoretic questions to be settled about it.

Probably the most important one is to determine which cardinals can appear as the covering number of this ideal. In $\mathbb{R}^{2}$ the covering number of $\mathcal{I}$ on a closed (or $G_{\delta}$ ) set behaves like the cardinality of the set: if it is uncountable then it is equal to the continuum. It is perhaps surprising that the behavior of $\gamma$ on closed sets in higher dimensions resembles the behavior of the meager ideal. The convexity number of the $\mathbb{R}^{3}$ set which is constructed in Theorem 21 can be made any prescribed uncountable cardinal $\kappa \leq 2^{\aleph_{0}}$ in a ccc forcing extension. 
In this section we provide a complete proof that $\gamma(S)$ for a closed $S \subseteq \mathbb{R}^{3}$ is not decided by ZFC.

The proof of this theorem was sketched in [13] in an, apparently, very unsatisfactory way. The main part that is missing from the sketch in [13] is the proof of the countable chain condition. Several people have asked for the details of this proof, therefore the proof is provided here $\left({ }^{1}\right)$ so that it may be available for future reference.

Theorem 29 (Kojman, Perles, Shelah). Let $S$ be the subset of $\mathbb{R}^{3}$ which is constructed in the proof of Theorem 21. If ZFC is consistent then it is also consistent that $2^{\aleph_{0}}=\aleph_{2}$ and $\gamma(S)=\aleph_{1}$.

Proof. Let $Q$ be the following partially ordered set. An element $p$ of $Q$ is a finite partial function from $K(S)$ to $\mathbb{N}$ so that no almost equilateral triple in the domain of $p$ is mapped to a single value. The order relation is inclusion.

Lemma 30. For every $x \in K$ the set $Q_{x}=\{p \in Q: x \in \operatorname{dom} p\}$ is dense in $Q$.

Pr o o f. Let $x \in K$ be given and let $p \in Q$ be arbitrary. If $x \notin \operatorname{dom} p$, let $n \in \mathbb{N}-\operatorname{ran} p$. Define $p^{\prime}=p \cup\{\langle x, n\rangle\}$. Since $\{x\}=p^{\prime^{-1}}(n)$ and $p^{\prime-1}(m)=$ $p^{-1}(m)$ for all $m \neq n$, there is no almost equilateral triple in $p^{-1}(l)$ for all $l \in \mathbb{N}$ and therefore $p^{\prime} \in Q_{x}$.

LEMma 31. If $G \subseteq Q$ is directed and meets every dense set, then $f:=$ $\bigcup G$ is a function from $K$ to $\mathbb{N}$ so that $\operatorname{conv}\left(f^{-1}(n)\right) \subseteq S$ for every $n \in \mathbb{N}$.

Pr o of. Since $G$ is directed, $f$ is indeed a function. Also, since $G \cap Q_{x} \neq \emptyset$ for each $x \in K$, the domain of $f$ is $K$. No almost equilateral triple exists in $f^{-1}(n)$ for all $n \in \mathbb{N}$ by the definition of $Q$. Since $S$ is simply connected, the convex hull of any subset of $S$ is contained in $S$ if and only if the convex hull of every 3-tuple from the subset is. Since a 3-tuple from $K$ is defected in $S$ iff it is almost equilateral, the proof is complete.

Lemma 32. $Q$ satisfies the countable chain condition.

Proof. Suppose to the contrary that $A=\left\{p_{\alpha}: \alpha<\omega_{1}\right\}$ is an anti-chain, that is, for no two members of $A$ is there a common extension in $Q$. For every $\alpha<\omega_{1}$ define a finite function $r_{\alpha}$ as follows. Let $x_{0}^{\alpha}, \ldots, x_{n-1}^{\alpha}$ enumerate $\operatorname{dom} p_{\alpha}$ and choose an open rational ball $u_{i}^{\alpha} \ni x_{i}^{\alpha}$ for each $i<n$ so that $x_{i}^{\alpha} \in u_{i}^{\alpha},\left\{u_{i}^{\alpha}: i<n\right\}$ are pairwise disjoint and the radius of each $u_{i}^{\alpha}$ is smaller than $\min \left\{d\left(x_{i}^{\alpha}, x_{j}^{\alpha}\right): i<j<n\right\} / 100$ and is, in addition, so small that for all $i_{0}<i_{1}<i_{2}<n$ either for every choice of $y_{j} \in u_{i_{j}}^{\alpha}$ the triple $\left\{y_{i_{0}}, y_{i_{1}}, y_{i_{2}}\right\}$ is almost equilateral or for every such choice $\left\{y_{i_{0}}, y_{i_{1}}, y_{i_{2}}\right\}$ is

$\left({ }^{1}\right)$ By courtesy of the editor. 
not almost equilateral. Now let $r_{\alpha}:\left\{u_{i}: i<n^{\alpha}\right\} \rightarrow \mathbb{N}$ be the finite function defined by $r_{\alpha}\left(u_{i}^{\alpha}\right)=p_{\alpha}\left(x_{i}\right)$.

Since there are uncountably many elements in $A$ and only countably many finite functions from rational neighborhoods to $\mathbb{N}$, by shrinking $A$ it may be assumed that $r_{\alpha}=r$ for some fixed $r$ for all $\alpha<\omega_{1}$.

Let $\alpha<\beta<\omega_{1}$ be fixed and a contradiction will be reached once it is seen that $p_{\alpha} \cup p_{\beta} \in Q$. If $x \in \operatorname{dom} p_{\alpha} \cup \operatorname{dom} p_{\beta}$ then $p_{\alpha}(x)=r(u)=p_{\beta}(x)$, where $u \in \operatorname{dom} r$ satisfies $x \in u$ Thus $p_{\alpha} \cup p_{\beta}$ is a function.

Let $\left\{x_{0}, x_{1}, x_{2}\right\} \subseteq \operatorname{dom}\left(p_{\alpha} \cup p_{\alpha}\right)$ be almost equilateral. Since at least two of the points from $\left\{x_{0}, x_{1}, x_{2}\right\}$ belong to the domain of either $p_{\alpha}$ or $p_{\beta}$, assume without loss of generality that $x_{0}, x_{1} \in \operatorname{dom} p_{\alpha}$. Therefore, $x_{0}$ and $x_{1}$ belong to different members of $\operatorname{dom} r$, say to $u_{0}, u_{1}$ respectively. Since $\left\{x_{0}, x_{1}, x_{2}\right\}$ is almost equilateral, $x_{2} \notin u_{0} \cup u_{1}$, and therefore $x_{2} \in u_{2}$ for some $u_{2} \in \operatorname{dom} r-\left\{u_{0}, u_{1}\right\}$. Let $x_{2}^{\prime}$ be the member of $\operatorname{dom} p_{\alpha}$ in $u_{2}$, so $p_{\alpha}\left(x_{2}^{\prime}\right)=$ $r\left(u_{2}\right)=\left(p_{\alpha} \cup p_{\beta}\right)\left(x_{2}\right)$. Since $\left\{x_{0}, x_{1}, x_{2}^{\prime}\right\} \subseteq \operatorname{dom} p_{\alpha}$ is almost equilateral, $r\left(u_{0}\right), r\left(u_{1}\right), r\left(u_{2}\right)$ are not equal to each other. Therefore, $\left\{x_{0}, x_{1}, x_{2}\right\}$ are not mapped by $p_{\alpha} \cup p_{\beta}$ to a single value.

Let $V$ be a universe of set theory which satisfies $2^{\aleph_{0}}=\aleph_{2}$. By the lemmas above, forcing with $Q$ over $V$ produces a cardinal preserving forcing extension $V^{Q}$ of $V$ in which $K^{V}$ is covered by countably many convex subsets of $S$. Since the rank $\varrho$ is absolute between transitive models of ZFC, the points of $S$ preserve their $V$ rank in the extension $V^{P}$. However, new members of $K$ may exist in $V^{P}$.

Let $R=\left\langle R_{\alpha}, Q_{\alpha}: \alpha<\omega_{1}\right\rangle$ be the finite support iteration of length $\omega_{1}$ in which $Q_{\alpha}$ is the partially ordered set $Q$ defined above, in $V^{R_{\alpha}}$. Since a finite support iteration of ccc forcing is ccc, $V^{R}$ is a cardinal preserving forcing extension of $V$ and hence satisfies $2^{\alpha_{0}}=\alpha_{2}$. For every $\alpha<\omega_{1}$, the set $K^{V^{R \alpha}}$ is covered by countably many convex subsets of $S$ in $V^{R_{\alpha+1}}$. Since $K^{R}=\bigcup_{\alpha<\omega_{1}} P^{V^{R_{\alpha}}}$, it follows that $K^{V^{R}}$ is the union of $\omega_{1}$ convex subsets of $S$. All other points of $S$ have rank at most 1 and are covered by countably many convex subsets of $S$. Thus $\gamma(S)=\alpha_{1}$ in $V^{R}$. This completes the proof of Theorem 29.

7. Concluding remarks and problems. The coarse classification of sets by their convexity number has several different refinements, obtained by ranking special subsets: In $\mathbb{R}^{2}$, closed, finitely convex sets are ranked by sizes of their visually independent subsets, and closed, countably convex sets by the Cantor degree of their 3-cliques. In a separable Banach space of dimension $>2$, closed sets are ranked by $\varrho$ which uses semi-cliques. There are other feasible means of classification one can apply: the dimension of a set can be defined as the least $n$ so that the chromatic number of $H^{n}(S)$ 
is $\gamma(S)$. This is always defined for subsets of a finite-dimensional space, and is defined for some sets in infinite-dimensional spaces, but it is easy to construct sets in an infinite-dimensional space, in the spirit of the set in Section 5, which are uncountably convex yet with countable chromatic number of $H^{n}(S)$ for each $n$.

The main problem which remains open is the classification of countably convex Borel sets. At the moment, this problem looks difficult. Perhaps the class of hypergraphs which are realized as convexity hypergraphs of Borel set is too broad and contains pathological examples. If this is so, then no classification of Borel sets will be possible. But it is not clear whether this is the case.

Another related problem is the complexity of the collection of all countably convex subsets of a given compact set in a Euclidean space. One can regard the collection of all closed subsets of, say, the unit sphere in $\mathbb{R}^{3}$ as a Polish space when endowed with the Hausdorff metric. It is not clear what the complexity of the collection of all countably convex closed subsets may be. The source of difficulty is, of course, the fact that countably convex subsets do not form an ideal, so the methods used in [10] and [9], Chapter VI $\left({ }^{2}\right)$, do not apply to this problem.

A particularly intriguing open problem concerning closed sets is the following:

Problem 33. Do closed subsets of $\mathbb{R}^{3}$ for which it is not consistent that their convexity number is smaller than the continuum coincide with those sets which contain a perfect clique? (The convexity number of a closed set which contains a perfect clique is always equal, of course, to the continuum.)

At any rate, the study of countable convexity leads to some interesting problems in descriptive set theory and perhaps will also be useful for Banach space geometry.

\section{References}

[1] M. Breen, A decomposition theorem for m-convex sets, Israel J. Math. 24 (1976), 211-216.

[2] - An $R^{d}$ analogue of Valentine's theorem on 3-convex sets, ibid., 206-210.

[3] M. Breen and D. C. Kay, General decomposition theorems for $m$-convex sets in the plane, ibid., 217-233.

[4] G. Cantor, Ueber unendliche, lineare Punktmannichfaltigkeiten, Nr. 6, Math. Ann. 23 (1884), 453-488.

[5] M. M. Day, Strict convexity and smoothness, Trans. Amer. Math. Soc. 78 (1955), $516-528$.

$\left({ }^{2}\right)$ I wish to thank an anonymous referee for pointing out this reference to me. 
[6] H. G. Eggleston, A condition for a compact plane set to be a union of finitely many convex sets, Proc. Cambridge Philos. Soc. 76 (1974), 61-66.

[7] V. Fonf and M. Kojman, On countable convexity of $G_{\delta}$ sets, in preparation.

[8] D. C. Kay and M. D. Guay, Convexity and a certain property $P_{m}$, Israel J. Math. 8 (1970), 39-52.

[9] A. S. Kechris and A. Louveau, Descriptive Set Theory and the Structure of Sets of Uniqueness, London Math. Soc. Lecture Note Ser. 128, Cambridge Univ. Press, 1987.

[10] A. S. Kechris, A. Louveau and W. H. Woodin, The structure of $\sigma$-ideals of compact sets, Trans. Amer. Math. Soc. 301 (1987), 263-288.

[11] V. Klee, Dispersed Chebyshev sets and covering by balls, Math. Ann. 257 (1981), 251-260.

[12] M. Kojman, Cantor-Bendixson degrees and convexity in $\mathbb{R}^{2}$, Israel J. Math., in press.

[13] M. Kojman, M. A. Perles and S. Shelah, Sets in a Euclidean space which are not a countable union of convex subsets, Israel J. Math. 70 (1990), 313-342.

[14] J. F. Lawrence, W. R. Hare, Jr. and J. W. Kenelly, Finite unions of convex sets, Proc. Amer. Math. Soc. 34 (1972), 225-228.

[15] A. J. Lazar and J. Lindenstrauss, Banach spaces whose duals are $L_{1}$ spaces and their representing matrices, Acta Math. 126 (1971), 165-194.

[16] J. Lindenstrauss and R. R. Phelps, Extreme point properties of convex bodies in reflexive Banach spaces, Israel J. Math. 6 (1968), 39-48.

[17] J. Matoušek and P. Valtr, On visibility and covering by convex sets, ibid. 113 (1999), 341-379.

[18] A. A. Milyutin, Ismorphisms of the spaces of continuous functions over compact sets of cardinality of the continuum, Teor. Funktsiu Funktsional. Anal. i Prilozhen. 2 (1966), 150-156.

[19] M. A. Perles and S. Shelah, A closed $n+1$-convex set in $R^{2}$ is the union of $n^{6}$ convex sets, Israel J. Math. 70 (1990), 305-312.

[20] F. A. Valentine, A three point convexity property, Pacific J. Math. 7 (1957), $1227-1235$.

Department of Mathematics and Computer Science

Ben Gurion University of the Negev

Beer Sheva, Israel

E-mail: kojman@math.bgu.ac.il

Received 7 June 1999;

in revised form 1 February 2000 